Journal of Circuits, Systems, and Computers, Vol. 8, Nos. 5 \& 6 (1998) 661-664

(C) World Scientific Publishing Company

\title{
AUTHOR INDEX \\ VOLUME 8 (1998)
}

Agarwal, V. K., see Thulasiraman, K.

8 (1998) 473-481

Ahmadi, M., see Djahanshahi H.

8 (1998) 447-451

Ahmadi, M., see Djahanshahi, $\mathrm{H}$.

Alla, H. and David R., Continuous and Hybrid Petri Nets

8 (1998) 589-604

Anastassopoulos, V., see Pneumatikakis, A. G.

8 (1998) $159-188$

Anderson, D., see Palusinski, O. A.

8 (1998) 461-472

8 (1998) 337-353

Anderson, H., see Palusinski, O. A.

Bi, G. and Chen, Y., Fast DHT Algorithms for Composite Sequence

Lengths

Bi, G., Kot, A. C., and Meng, Z., Generalised Split-Radix Algorithms for DFT

Bobbio, A., Puliafito, A., Telek, M., and Trivedi, K. S., Recent

Developments in Non-Markovian Stochastic Petri Nets

8 (1998) $337-353$

8 (1998) 421-434

8 (1998) 405-409

8 (1998) 119-158

8 (1998) $267-272$

Card H. C., McNeill, D. K., Schneider, C. R., Schneider, R. S., and

Dolenko, B. K., Tolerance of On-Chip Learning To Various Circuit Inaccuracies

Card, H. C., see Rosendahl, G. K.

Card, H., Artificial Neural Computations in Digital Arrays

Cauwenberghs, G., see Lubkin, J.

Chen, Y., see $\mathrm{Bi}, \mathrm{G}$.

Chia, C. Y., see Tan, E. C.

Constantinides, A. G., see Pneumatikakis, A. G.

Cortadella, J., see Kondratyev A.

Das, A., see Thulasiraman, K.

David R., see Alla, H.

Deliyannis, T., see Pneumatikakis, A. G.

Dimitrov, V. S., see Sadeghi-Emamchaie, S.

Djahanshahi H., Ahmadi, M., Jullien, G. A., and Miller, W. C.,

A Low-Variation Nonlinear Neuron Circuit

8 (1998) 315-327

8 (1998) $453-459$

8 (1998) $525-539$

8 (1998) 605-614

8 (1998) $421-434$

8 (1998) 355-362

8 (1998) $461-472$

8 (1998) $67-118$

8 (1998) $473-481$

8 (1998) $159-188$

8 (1998) $461-472$

8 (1998) 615-635

8 (1998) 447-451

Djahanshahi, H., Ahmadi, M., Jullien, G. A., and Miller, W. C.,

Neural Network Integrated Circuits with Single-Block Mixed Signal Arrays

Dolenko, B. K., see Card H. C.

Erten, G. and Salam, F. M., Two Cellular Architectures for Integrated Image Sensing and Processing on a Single Chip

Erten, G., see Salam, F. M.

Gaudet, V. C. and Gulak, P. G., Implementation Issues for High-Bandwidth Field-Programmable Analog Arrays

Gettman, D. M., see Palusinski, O. A.

8 (1998) 589-604

8 (1998) 315-327

8 (1998) 637-659

8 (1998) $571-587$

8 (1998) $541-558$

8 (1998) $337-353$ 
Gitau, M. N., Smith, I. R., and Kettleborough, J. G., Mathematical Modeling of Analog Controlled Voltage Source Converters for Improved Dynamic Response

Gulak, P. G., see Gaudet, V. C.

Huang J. S. and Murata T., A Constructive Method for Finding Legal Transition Sequences in Petri Nets

Huang, K. Y., see Thulasiraman, K.

Jin, L. W. and Wei, G., Handwritten Chinese Character Recognition with Directional Decomposition Cellular Features

Jullien, G. A., see Djahanshahi H.

Jullien, G. A., see Djahanshahi, $\mathrm{H}$.

Jullien, G. A., see Sadeghi-Emamchaie, S.

Kettleborough, J. G., see Gitau, M. N.

Kishinevsky, M., see Kondratyev A.

Kondratyev A., Kishinevsky, M., Taubin, A., Cortadella, J., and Lavagno L., The Use of Petri Nets for the Design and Verification of Ansynchronous Circuits and Systems

Kot, A. C., see Bi, G.

Kung, L. Y., see Wang, W. F.

Lai, Y. M., Tse, C. K., and Mehta, P., A Symbolic Circuit Modeling Program for DC/DC PowerConverter Circuits

Lam, H. K., see Leung, F. H. F.

Lavagno L., see Kondratyev A.

Lee, T. S., A CMOS Low-Voltage Transconductor for VHF Continuous-Time Filters

Leung, F. H. F., Wong, L. K., Tam, P. K. S., and Lam, H. K., Realization of Analog Fuzzy Logic Control for PWM Boost Converters

Liang, Q.-H. and Zhu, M.-L., A Combined Approach to Text-Dependent Speaker Identification: Comparison with Pure Neural Net Approaches

Lubkin, J. and Cauwenberghs, G., A Learning Parallel

Analog-To-Digital Vector Quantizer

Marcjan, C., see Palusinski, O. A.

McLeod, R. D., see Rosendahl, G. K.

McNeill, D. K., see Card H. C.

Mehta, P., see Lai, Y. M.

Meng, Z. see Bi, G.

Miller, W. C., see Djahanshahi H.

Miller, W. C., see Djahanshahi, H.

Miller, W. C., see Sadeghi-Emamchaie, S.

8 (1998) 483-496

8 (1998) 541-558

8 (1998) 189-222

8 (1998) 473-481

8 (1998) 517-524

8 (1998) 447-451

8 (1998) 589-604

8 (1998) 615-635

8 (1998) 483-496

8 (1998) 67-118

8 (1998) 67-118

8 (1998) 405-409

8 (1998) 379-404

8 (1998) 329-335

8 (1998) 411-419

$8(1998) 67-118$

8 (1998) 497-505

8 (1998) 411-419

8 (1998) 273-281

8 (1998) 605-614

8 (1998) 337-353

8 (1998) $453-459$

8 (1998) 315-327

8 (1998) $329-335$

8 (1998) 405-409

8 (1998) $447-451$

8 (1998) 589-604

8 (1998) 615-635

Milovanović, D. Bojković, Z., and Samčovic, A. Upper Bound on SNR

Gain in Wavelet Transform Predictive-Entropy Image Coding

8 (1998) $267-272$

Mueller S. M. and Paul, W. J., On the Correctness of Hardware

Scheduling Mechanisms for Out-of-Order Execution

8 (1998) 301-314

8 (1998) $189-222$

8 (1998) 283-299

Murthy, K. V. V., see Pai, K. R.

8 (1998) 559-569

Newcomb, R. W., see Sellami, L.

Newman, A., Shatz, S. M., and Xie X., An Approach to Object

System Modeling by State-Based Object Petri Nets

8 (1998) 1-20 
Pai, K. R., Murthy, K. V. V., and Ramachandran, V.,

Chebyshev-Family of Transitional Filters

Palusinski, O. A., Gettman, D. M., Anderson, D., Anderson, H., and Marcjan, C., Filtering Applications of Field Programmable Analog Arrays

Paul, W. J., see Mueller, S. M.

Pneumatikakis, A. G., Constantinides, A. G., Deliyannis, T., and Anastassopoulos, V., Sigma-Delta IIR Filter Realization Using Third-Order Remodulators

Puliafito, A., see Bobbio, A.

Ramachandran, V., see Pai, K. R.

Rasmussen, A., see Sellami, L.

Recalde, L., Teruel, E., and Silva M., On Linear Algebraic Techniques for Liveness Analysis of $P / T$ Systems

8 (1998) 283-299

8 (1998) 337-353

8 (1998) 301-314

8 (1998) 461-472

8 (1998) 119-158

8 (1998) 283-299

8 (1998) 559-569

Rosendahl, G. K., McLeod, R. D., and Card, H. C., A DSP-FPGA-Based Reconfigurable Computer

8 (1998) 223-265

8 (1998) $453-459$

Sadeghi-Emamchaie, S., Jullien, G. A., Dimitrov, V. S., and Miller, W. C., Digital Arithmetic Using Analog Cellular Neural Networks

Salam, F. M., see Erten, G.

Salam, F. M., Wang, Y., and Erten, G. Analog Dendro-Dendritic Arrays with Digital On-Chip Learning

Salama, K. N. and Soliman, A. M. Active RC Filters Using Operational Transresistance Amplifiers

Samčovic, A., see Milovanović, D.

Schneider, C. R., see Card H. C.

Schneider, R. S., see Card H. C.

Sellami, L., Singh, S. K., Newcomb, R. W., Rasmussen, A., and Zaghloul, M. E., VLSI Floating Resistors for Neural Type Cell Arrays

Shatz, S. M., see Newman, A.

Silva M., see Recalde, L.

Singh, S. K., see Sellami, L.

Smith, I. R., see Gitau, M. N.

Soliman, A. M., New Grounded-Capacitor Current-Mode Oscillators Using Single-Output CCIIS

Soliman, A. M., see Salama, K. N.

Tam, P. K. S., see Leung, F. H. F.

Tan, E. C. and Chia, C. Y., Interpolation of Multiplier Coefficients of an Elliptic Wave Digital Filter

Taubin, A., see Kondratyev A.

Telek, M., see Bobbio, A.

Teruel, E., see Recalde, L.

Thulasiraman, K., Das, A., Huang, K. Y., and Agarwal, V. K., Correct Diagnosis of Almost All Faulty Units in a Multiprocessor System

Trivedi, K. S., see Bobbio, A.

Tse, C. K., see Lai, Y. M.

van der Aalst, W. M. P., The Application of Petri Nets to Workflow Management

8 (1998) 615-635

8 (1998) 637-659

8 (1998) 571-587

8 (1998) 507-516

8 (1998) 267-272

8 (1998) 315-327

8 (1998) 315-327

8 (1998) 559-569

8 (1998) 1-20

8 (1998) 223-265

8 (1998) 559-569

8 (1998) 483-496

8 (1998) 363-378

8 (1998) 507-516

8 (1998) 411-419

8 (1998) 355-362

8 (1998) 67-118

8 (1998) 119-158

8 (1998) 223-265

8 (1998) 473-481

8 (1998) 119-158

8 (1998) 329-335

8 (1998) 21-66 
Wang, W. F. and Kung, L. Y., On a Refined Cyclic

Reservation-Based Mac Scheme for Ultra High-Speed Hierarchical Ring Mans

Wang, Y., see Salam, F. M.

Wei, G., see Jin, L. W.

Wong, L. K., see Leung, F. H. F.

Xie X., see Newman, A.

Yan, J.-T., Routing Space Estimation and Assignment for Macro-Cell Placement

Zaghloul, M. E., see Sellami, L.

Zhu, M.-L., see Liang, Q.-H.

8 (1998) 379-404

8 (1998) 571-587

8 (1998) 517-524

8 (1998) 411-419

8 (1998) $1-20$

8 (1998) 435-446

8 (1998) 559-569

8 (1998) 273-281 